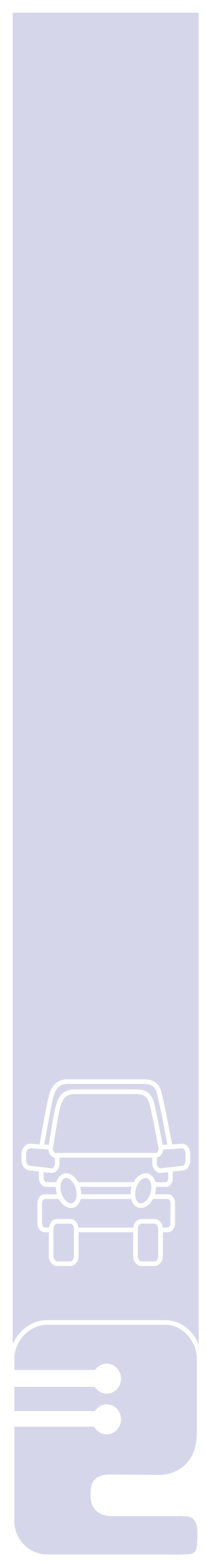

\title{
Control Predictivo con Restricciones de Suspensión Vehicular Activa
}

\author{
Carlos Arturo Giraldo Gutiérrez ${ }^{1}$
}

Recibido: Junio 16 de 2013 Aprobado: Octubre 29 de 2013

\section{Resumen:}

Las vibraciones y perturbaciones que se presentan en el chasis de los vehículos al desplazarse sobre terrenos irregulares pueden ser atenuadas mediante el uso de suspensiones activas que modifiquen la energía del sistema; en este artículo se exponen los resultados del diseño y simulación de una suspensión activa, utilizando el modelo clásico de masas suspendidas y la técnica de control predictivo basado en modelo, mediante comparación de simulaciones del comportamiento de los sistemas de suspensión pasivo y activo con control predictivo. Los resultados evidencian una mejora significativa en el desempeño de la suspensión al rechazar las perturbaciones del terreno.

Palabras clave: Suspensión Vehicular Activa, Modelamiento Matemático, Control Predictivo, MATLAB, Simmechanics.

Abstract:

The vibrations and shocks that occur on the chassis of the vehicle when traveling over rough terrain can be mitigated through the use of active suspensions which modify the energy of the system; in this article the results of the design and simulation of an active suspension are presented, using the classical model of suspended masses and the technique of predictive control based on model, by comparing simulations of the behavior of passive suspension systems and active ones with predictive control. The results show a significant improvement in the performance of the suspension to reject the disturbances of the terrain.

Keywords: Vehicular active suspension, mathematical modeling, predictive control, MATLAB, Simmechanics.

1 Ingeniero Electrónico, Director del programa de Tecnología en Electrónica, Facultad de IngenieríaUNIMINUTO. 


\section{INTRODUCCIÓN}

El control predictivo es una técnica moderna de control moderno que permite diseñar controladores óptimos minimizando parámetros deseados, teniendo en cuenta las restricciones físicas impuestas por el sistema o las restricciones requeridas por el proceso o el usuario. Debido a la formulación del control predictivo como un problema de optimización, permite trabajar el sistema controlado cerca de las restricciones del mismo, obteniendo el mejor desempeño posible de acuerdo con la función objetivo y las restricciones definidas (Maciejowski, 2000). Teniendo en cuenta las características del control predictivo se ha incrementado su aplicación en procesos industriales y en investigaciones que hacen uso de esta técnica para obtener el mayor provecho de sus beneficios en diferentes aplicaciones, entre las cuales están, control de nivel de tanques acoplados (Khalid \& Kadri, 2012), control de fuentes de energía (Hredzak, Agelidis, \& Jang, 2013) y el control de trafico vehicular (Kamal, Imura, Hayakawa, Ohata, \& Aihara, 2013).

Las características del control predictivo permiten aplicarlo a los sistemas de suspensión vehicular, en donde se tendrán en cuenta las restricciones físicas impuestas por la disposición de los componentes y las limitaciones de los actuadores, así como los parámetros a minimizar que definen el comportamiento ante perturbaciones en la vía. Al mejorar el desempeño de la suspensión, el vehiculo responderá mas rápido a los cambios en la superficie de desplazamiento, incrementando la seguridad y el confort en el transporte de pasajeros y carga.

\section{PLANTEAMIENTO DEL PROBLEMA}

Los vehículos se diseñan con el objetivo de proveer un viaje confortable a los pasajeros o para mantener la integridad de la carga que se transporta. La suspensión del vehículo reduce el efecto que tienen las imperfecciones de la vía sobre el movimiento del chasis, pero la capacidad de reducirlas se encuentra directamente relacionada con los parámetros físicos que describen el comportamiento de los elementos que conforman el sistema de suspensión. Dependiendo de la velocidad del vehículo y de la perturbación presente en la vía, existe la posibilidad que la suspensión no realice adecuadamente su trabajo y se experimenten movimientos desagradables para los pasajeros y en el peor de los casos se ponga en riesgo su integridad. Algunas cargas transportadas no pueden someterse a movimientos en donde se experimenten cambios repentinos de aceleración vertical, ya que podrían destruir la carga. Por lo anterior se requiere de un sistema que tenga la capacidad de reaccionar rápidamente a las perturbaciones de la vía mediante la inyección o sustracción de energía del sistema.

\section{METODOLOGÍA}

El trabajo realizado se desarrolló en tres fases, Modelamiento matemático, Diseño del controlador MCP y Comparación comportamental, las cuales se describen a continuación:

3.1 Determinar el modelo matemático del sistema de masas suspendidas a partir de las ecuaciones dinámicas y su comprobación mediante simulaciones en MATLAB y Simmechanics. Se compararán las respuestas del modelo mecánico simulado en Simmechanics con las respuestas del modelo matemático, comprobando oscilaciones y tiempos de establecimiento.

3.2 Diseño del controlador predictivo basado en modelo, teniendo en cuenta las restricciones físicas impuestas por el sistema, tales como fuerza máxima ejercida por el actuador y su máxima tasa de cambio. Al tener en cuenta estas limitaciones en el proceso de optimización no se enviarán ordenes al actuador que excedan sus condiciones de operación y el sistema intentará reducir la función objetivo, conociendo las limitaciones del actuador.

3.3 Comparar mediante simulaciones en MATLAB los comportamientos de las suspensiones pasiva y activa con control MCP (Model Predictive Control) al presentarse perturbaciones en la superficie de desplaza-miento. Se realizarán simulaciones con perturba-ciones estandar y se compararán las respuestas en desplazamiento, velocidad y aceleración de los elementos de la suspensión (Chasis y Rueda).

\section{PROCEDIMIENTO}

\subsection{Modelamiento Matemático}

El control predictivo basado en modelo hace uso del estado actual del sistema a controlar y de su modelo 
matemático, para predecir el comportamiento del sistema en un intervalo de tiempo con el objetivo de determinar las acciones a realizar por los actuadores que minimizen una función objetivo establecida en el diseño del controlador. La función objetivo contempla las variables que se desean minimizar, que en el caso de la suspensión vehicular son aceleración, velocidad y posición. La acción realizada por el actuador en el caso específico de la suspensión activa vehicular es la aplicación de fuerzas entre los elementos que la componen. Si el modelo de predicción del sistema no lo representa de manera adecuada, la predicción será erronea y las acciones realizadas por el controlador MCP no tendrán el efecto deseado en la minimización de la función objetivo establecida.

Se han desarrollado modelos matemáticos que tienen en cuenta la geometría de los diferentes tipos de suspensiones vehiculares independientes como MacPherson y Doble Triangulo (M. S. Fallah, 2008), (Keum-Shik Hong, 1999), obteniendo dinámicas más precisas con respecto al comportamiento de los sistemas reales, sin embargo el modelo de dos masas suspendidas es más simple y sus resultados son aceptables para modelar el comportamiento de suspensiones independientes.

A continuación se obtienen las ecuaciones que definen la dinámica del sistema incluyendo la acción del actuador. En la Figura 1 se ilustra el modelo de masas suspendidas utitilizado para representar la suspensión vehicular.

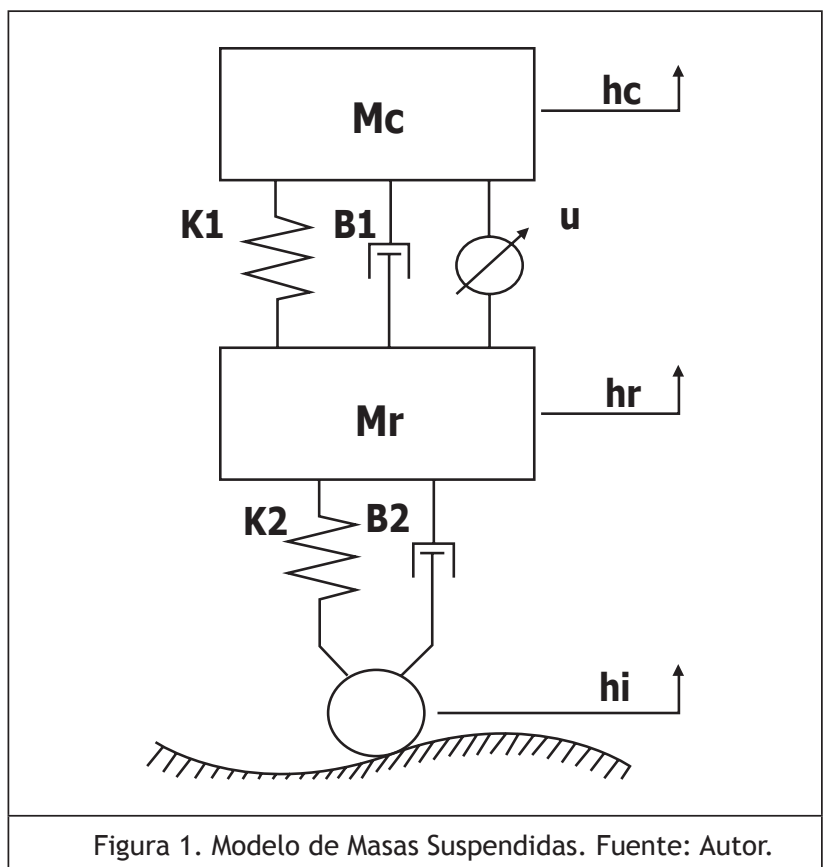

En el modelo de la Figura 1, las constantes indicadas representan:

\begin{tabular}{|c|l|}
\hline \multicolumn{2}{|c|}{ Tabla 1. Parámetros usados en el modelo de suspensión de } \\
masas suspendidas. \\
\hline Mc & Masa del Chasis \\
\hline Mr & Masa de la Rueda \\
\hline K1 & $\begin{array}{l}\text { Constante Elástica del Resorte de la } \\
\text { Suspensión }\end{array}$ \\
\hline B1 & $\begin{array}{l}\text { Coeficiente de Fricción Viscosa del } \\
\text { Amortiguador de la Suspensión }\end{array}$ \\
\hline u & Fuerza Ejercida por el Actuador \\
\hline K2 & Constante Elástica de la Rueda \\
\hline B2 & Coeficiente de Fricción Viscosa de la Rueda \\
\hline hc & Desplazamiento Vertical del Chasis \\
\hline hr & Desplazamiento Vertical de la Rueda \\
\hline hi & $\begin{array}{l}\text { Desplazamiento Vertical de las } \\
\text { Perturbaciones en la Superficie }\end{array}$ \\
\hline
\end{tabular}

Partiendo del modelo de la Figura 1 y por medio de diagramas de cuerpo libre para cada una de las masas y las leyes de Newton, se obtienen las siguientes ecuaciones de movimiento:

$M c * h c=-B 1(h c-h r)-K 1(h c-h r)+u$

$M r * h r=B 1(h c-h r)+K 1(h c-h r)+B 2(h i-h r)+K 2(h i-h r)-u(2)$

Donde los énfasis superiores de las variables “.””, “’” indican segunda y primera derivada con respecto al tiempo.

Manipulando algebraicamente las ecuaciones anteriores se obtiene un sistema lineal de ecuaciones diferenciales de primer orden que se denomina representación en espacio de estados, donde las derivadas de las variables y las salidas del sistema estarán representadas por combinaciones ponderadas de variables sin derivar, a este grupo de variables se les denomina variables de estado (Ogata, 1998). Estos sistemas se representan usualmente mediante las siguientes relaciones matriciales:

$\dot{x}=A x+B u$

$y=C x+D u$

Con el fin de obtener un modelo en espacio de estados en donde requiera como entrada el estímulo del actuador y la perturbación sobre la vía $h i$, de tal manera que la derivada $h i$ se encuentre establecida por la dinámica del sistema, se definen las siguientes variables de estado: 
$\begin{array}{ll}x_{1}=M r * h r-B 2(h i-h r) & (5) \\ x_{2}=h r & (6) \\ x_{3}=h c & (7) \\ x_{4}=h c & \text { (8) }\end{array}$

Manipulando algebraicamente las ecuaciones (1) y (2), y sustituyendo (5), (6), (7) y (8), se obtiene el siguiente modelo en espacio de estados:

$\left[\begin{array}{c}\dot{x}_{1} \\ \dot{x}_{2} \\ \dot{x}_{3} \\ \dot{x}_{4}\end{array}\right]=\left[\begin{array}{cccc}\frac{-B 1}{M r} & \frac{B 1 * B 2}{M r}-K 1-K 2 & K 1 & B 1 \\ \frac{1}{M r} & \frac{-B 2}{M r} & 0 & 0 \\ 0 & 0 & 0 & 1 \\ \frac{B 1}{M c * M r} & \frac{-B 1 * B 2}{M c * M r}+\frac{K 1}{M c} \frac{-K 1}{M c} & \frac{-B 1}{M c}\end{array}\right]\left[\begin{array}{l}x_{1} \\ x_{2} \\ x_{3} \\ x_{4}\end{array}\right]+$

$\left[\begin{array}{cc}-1 & \mathrm{~K} 2-\frac{B 1 * B 2}{M r} \\ 0 & \frac{B 2}{M r} \\ 0 & 0 \\ \frac{1}{M c} & \frac{B 1 * B 2}{M c * M r}\end{array}\right]\left[\begin{array}{c}u \\ h i\end{array}\right]$

$\left[\begin{array}{l}y_{1} \\ y_{2} \\ y_{3} \\ y_{4}\end{array}\right]=\left[\begin{array}{cccc}0 & 0 & 1 & 0 \\ 0 & 0 & 0 & 1 \\ 0 & 1 & 0 & 0 \\ \frac{1}{M r} & \frac{-B 2}{M r} & 0 & 0\end{array}\right]\left[\begin{array}{l}x_{1} \\ x_{2} \\ x_{3} \\ x_{4}\end{array}\right]+\left[\begin{array}{cc}0 & 0 \\ 0 & 0 \\ 0 & 0 \\ 0 & \frac{B 2}{M r}\end{array}\right]\left[\begin{array}{c}u \\ h i\end{array}\right]$

(10)
Las salidas del modelo anterior $y_{1}, y_{2}, y_{3}, y_{4}$ corresponden respectivamente a posición del chasis $(h c)$, velocidad del chasis $(h c)$, posición de la rueda $(h r)$ y velocidad de la rueda $(h r)$.

El modelo obtenido se valida mediante el software MATLAB y la herramienta SIMULINK. En la figura 2 se muestra el modelo definido por las ecuaciones de estado y el modelo en Simmechanics de SIMULINK que determina el comportamiento de sistemas mecánicos. Los valores utilizados para las constantes del modelo se obtuvieron de (Jorge Hurel, 2012), los cuales son:

Tabla 2. Magnitudes usadas en el modelo de masas suspendidas. Fuente: (Jorge Hurel, 2012).

\begin{tabular}{|c|c|c|}
\hline Constante & Valor & Unidades \\
\hline Mc & 439.4 & $\mathrm{Kg}$ \\
\hline Mr & 42.3 & $\mathrm{Kg}$ \\
\hline K1 & 38404 & $\mathrm{~N} / \mathrm{m}$ \\
\hline B1 & 3593.4 & $\mathrm{Ns} / \mathrm{m}$ \\
\hline K2 & 310000 & $\mathrm{~N} / \mathrm{m}$ \\
\hline B2 & 3100 & $\mathrm{Ns} / \mathrm{m}$ \\
\hline
\end{tabular}

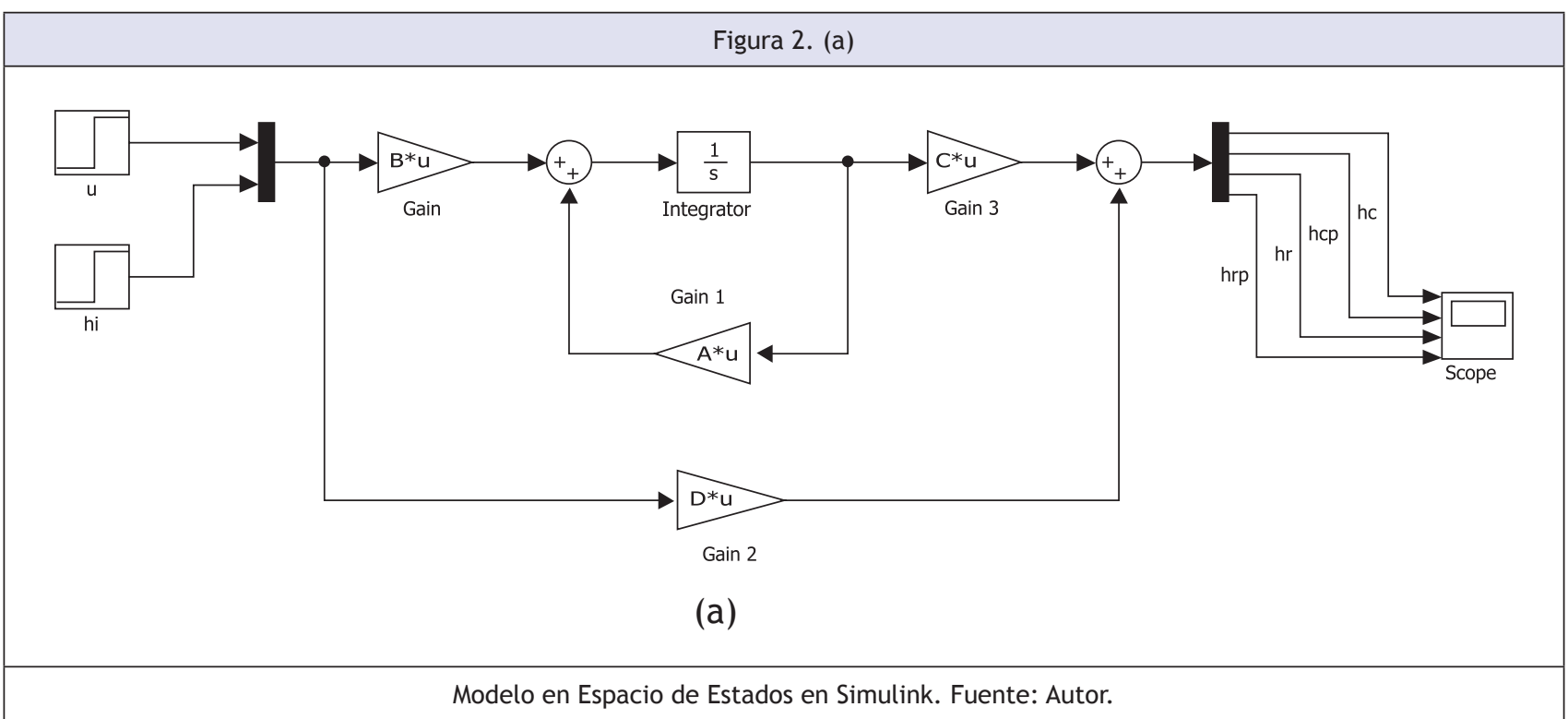




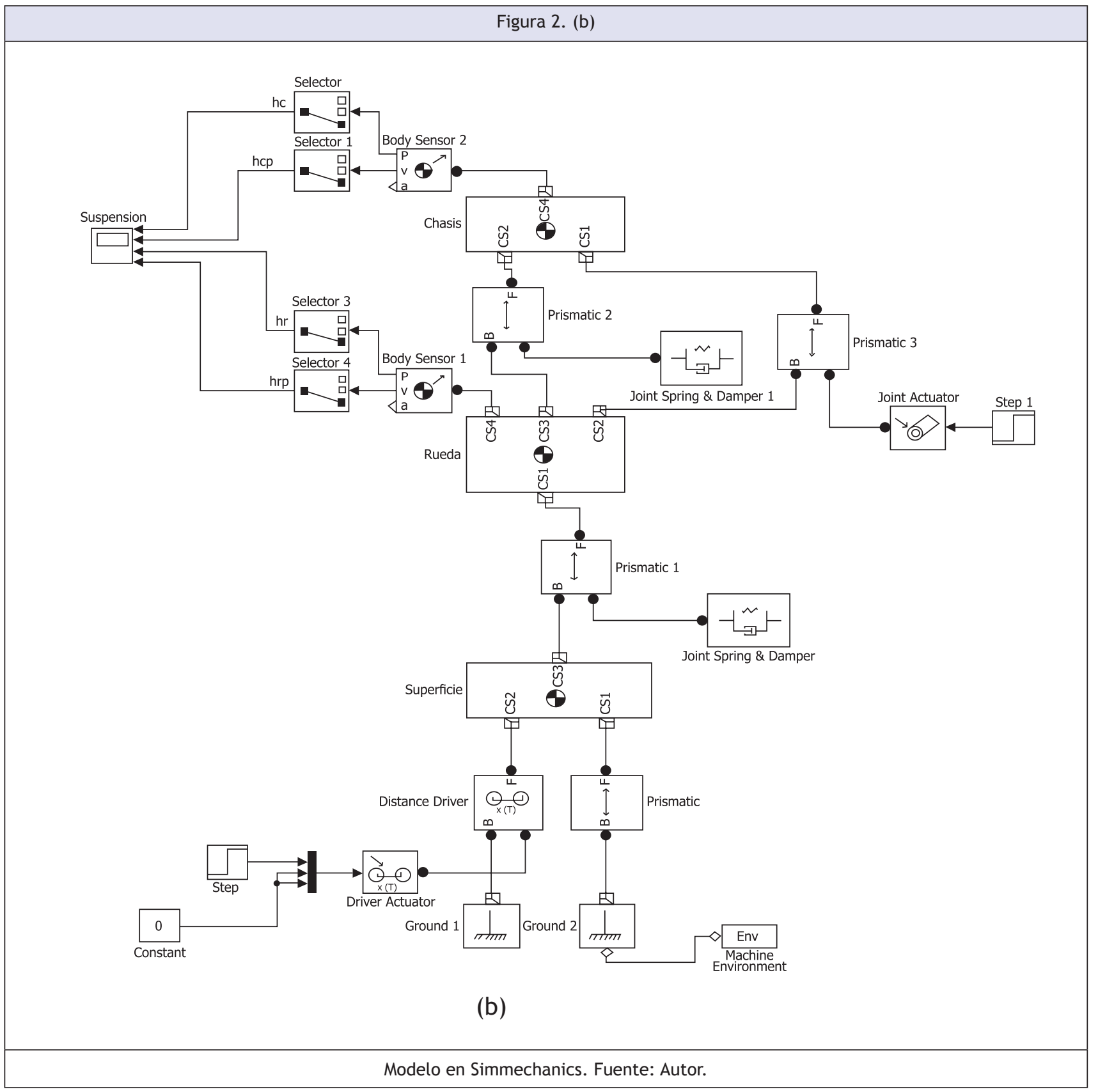

Las simulaciones que se desarrollarán sobre estos dos modelos se realizarán considerando una perturbación en la superficie de desplazamiento de forma escalón con $5 \mathrm{~cm}$ de amplitud.

\subsection{Diseño del Controlador MCP}

Es necesario realizar la digitalización del sistema a un tiempo de muestreo lo suficientemente corto como para que capture con precisión la dinámica del sistema continuo. La razón de la digitalización radica en la simplicidad de determinar el comportamiento futuro del sistema mediante el uso recursivo de datos de entrada y salida, evitando la necesidad de realizar integraciones, como es para sistemas continuos. En este caso se realiza una digitalización con un tiempo de muestreo de 10 ms usando la función de MATLAB $c 2 d$ (continuos to digital), obteniendo el siguiente modelo digital en espacio de estados: 


$$
\begin{aligned}
{\left[\begin{array}{l}
x_{1}(k+1) \\
x_{2}(k+1) \\
x_{3}(k+1) \\
x_{4}(k+1)
\end{array}\right]=} & {\left[\begin{array}{cccc}
399.5 e-3 & -432.7 & 242.1 & 24.0 \\
104.9 e-6 & 430.4 e-3 & 26.1 \mathrm{e}-3 & 2.5 \mathrm{e}-3 \\
5.8 e-6 & -21.4 e-3 & 996.5 e-3 & 9.6 e-3 \\
870.2 e-6 & -3.8 & -622.1 e-3 & 938.3 e-3
\end{array}\right]\left[\begin{array}{l}
x_{1}(k) \\
x_{2}(k) \\
x_{3}(k) \\
x_{4}(k)
\end{array}\right]+\left[\begin{array}{c}
-6.3 \mathrm{e}-3 \\
-681.4 e-9 \\
89.1 e-9 \\
16.2 e-6
\end{array}\right][u(K)] } \\
& {[y(K)]=\left[\begin{array}{llll}
19.3 e-3 & -51.8 & -8.0 & 182.2 e
\end{array}\right]\left[\begin{array}{c}
x_{1}(k) \\
x_{2}(k) \\
x_{3}(k) \\
x_{4}(k)
\end{array}\right]+[227.5 e-6][u(K)] }
\end{aligned}
$$

En el modelo anterior solo se tiene en cuenta la entrada del actuador del sistema, no se tiene en cuenta la entrada de perturbación, esto debido al desconocimiento de la señal de perturbación en el sistema de la suspensión. Esta señal podría tenerse en cuenta en el caso de utilizar un sistema de sensado y previsualización de las perturbaciones presentes en la superficie. La salida del modelo corresponde a la señal que se desea controlar, en este caso una ponderación de los parámetros de aceleración, velocidad y posición relativa del chasis.

El controlador predictivo opera haciendo uso del modelo digital del sistema, el estado actual de las variables de estado y algoritmos de optimización para determinar las señales de control a introducir al sistema que minimizan la función objetivo establecida en el diseño, teniendo en cuenta las restricciones impuestas sobre la entrada y la salida. En la figura 3 se ilustra la estructura del controlador predictivo basado en modelo.

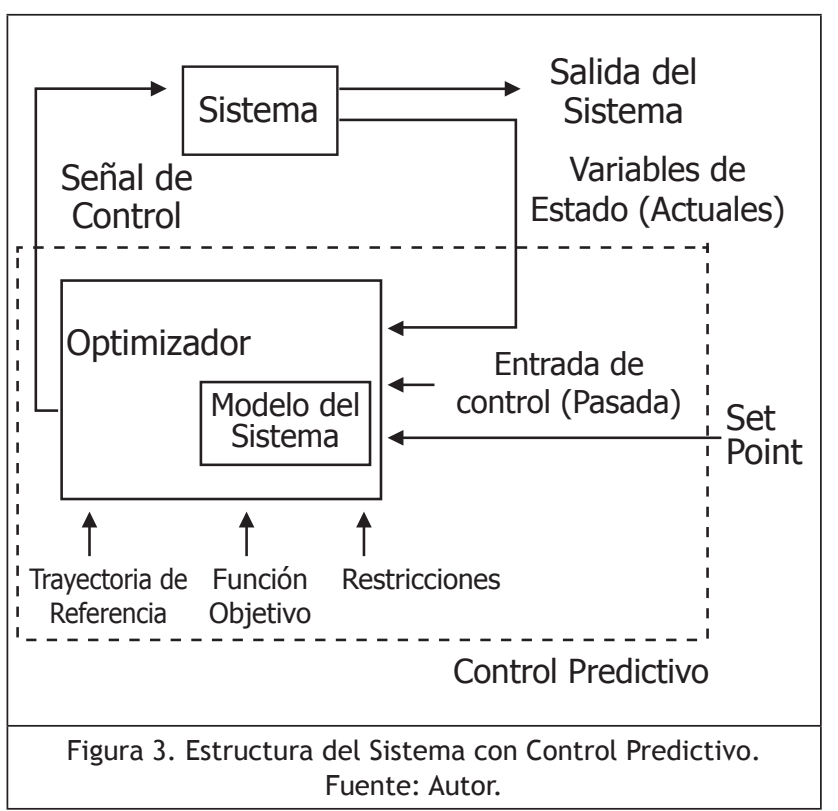

En la estructura ilustrada en la Figura 3 se encuentra la señal Trayectoria de Referencia, la cual es tenida en cuenta por el optimizador para determinar la señal de control que lleva el modelo del sistema siguiendo la trayectoria de referencia a alcanzar el valor establecido en el Set Point.

En el procedimiento de diseño se establecen varios parámetros que definen el comportamiento del controlador predictivo basado en modelo, los cuales son horizonte de predicción, horizonte de control y función objetivo. El horizonte de predicción establece cuantas muestras hacia el futuro tendrá en cuenta el optimizador, el horizonte de control establecerá el número de señales de control futuras que serán aplicadas en el proceso de predicción y optimización, y por último la función objetivo penalizará el seguimiento de la trayectoria hacia el Set Point y las variaciones en la señal de control.

El optimizador determinará los valores de la señal de control para instantes futuros establecidos en el horizonte de control sin violar las restricciones impuestas. A pesar de obtener varias entradas futuras dependiendo del horizonte de control, solamente se aplica al sistema la primera señal de control, a esta técnica se le denomina Horizonte Disminuido, la razón se encuentra en los posibles futuros cambios que puedan presentarse en el sistema real que no se encuentran modelados en el sistema utilizado en el optimizador, como perturbaciones presentes en los actuadores y sensores, de tal forma que en cada ciclo de control se predice y se optimiza la señal de control para el estado actual del sistema real.

En el caso particular del sistema de suspensión activo, se establecieron los siguientes parámetros para el controlador predictivo basado en modelo. (Tabla 3). 


\begin{tabular}{|l|ll|}
\hline \multicolumn{2}{|c|}{ Tabla 3. Parámetros del controlador predictivo basado en modelo (MPC). Fuente: Autor. } \\
\hline & $J=\left(R_{s}-Y\right)^{T}\left(R_{s}-Y\right)+\triangle U^{T} \bar{R} \triangle U$ \\
& Sujeto a: \\
& $M * \Delta U \leq y$ \\
& Donde: & \\
& $R_{s}$ & Trayectoria deseada de retorno a SetPoint. \\
& $Y$ & Señal a controlar de salida del sistema. \\
& $\triangle U$ & Cambios de la señal de Control. \\
& $\bar{R}$ & Matriz de penalización de la señal de control. \\
& $M, y$ & Son matrices que definen las restricciones. \\
\hline Función de Salida a Controlar & \multicolumn{1}{c}{$Y=0.1 *(h c)+h c+0.7 *(h c-h r)$} \\
\hline Horizonte de Predicción & \multicolumn{1}{c}{$H p=20$} \\
\hline Horizonte de Control & & $H c=10$ \\
\hline
\end{tabular}

Las restricciones impuestas son:

- La señal de control no puede tener variaciones superiores a $0.5 \mathrm{KN}$ entre intervalos adyacentes de tiempo, lo que equivale a un límite en la velocidad de respuesta del actuador de $50 \mathrm{KN} / \mathrm{s}$.

- La señal de control no puede superar los $2 \mathrm{KN}$ pico.

Las anteriores restricciones son impuestas por la velocidad de respuesta del actuador y los límites máximos. En este trabajo se suponen los límites de operación del actuador, pero en el caso de la aplicación sobre un sistema real estos valores serán establecidos por las limitaciones. Los valores anteriores son coherentes con la información suministrada en la literatura, se mantienen dentro de el orden de magnitud de actuadores usados en investigaciones previas, tal como el usado en (Bart L.J. Gysen, 2011).

\subsection{Comparación Comportamental}

Las simulaciones del sistema de suspensión pasiva y del sistema con control MCP se realizan mediante un script en MATLAB en donde mediante lineas de código se define el sistema en espacio de estados y se simula de manera recursiva, obteniendo iteración a iteración, la señal de control que minimiza la función objetivo establecida, así como las variables que permitirán realizar el análisis de su comportamiento. La perturbación en la superficie de desplazamiento será de forma escalón con $5 \mathrm{~cm}$ de amplitud, la cual permitirá observar la respuesta del sistema de acuerdo a comportamientos bien conocidos de los sistemas de segundo orden a entradas tipo paso. La amplitud de $5 \mathrm{~cm}$ ha sido utilizada dentro de la literatura, teniendo en cuenta que la suspensión convencional aproxima estructuras de suspensiones mas complejas como la Macpherson, DobleWisborn, Multilink, entre otras, por lo cual la amplitud se mantiene reducida para que las dinámicas de la suspensión de masas suspendidas se aproximen a dichas suspenciones cuando se presentan perturbaciones que no llevan al sistema a condiciones distantes de su punto de equilibrio.

\section{ANÁLISIS DE RESULTADOS}

En la figura 4, se muestran las respuestas obtenidas mediante simulación del sistema en espacio de estados y el modelo mecánico en Simmechanics.

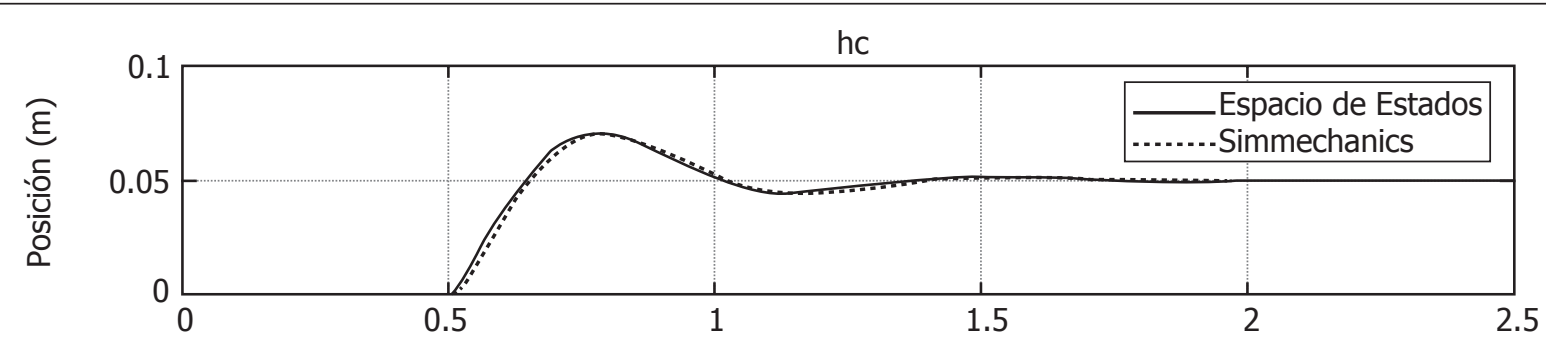

Figura 4. Respuestas de los modelos en Espacio de Estados y en Simmechanics a perturbación tipo escalón de 5cm. Fuente: Autor. 


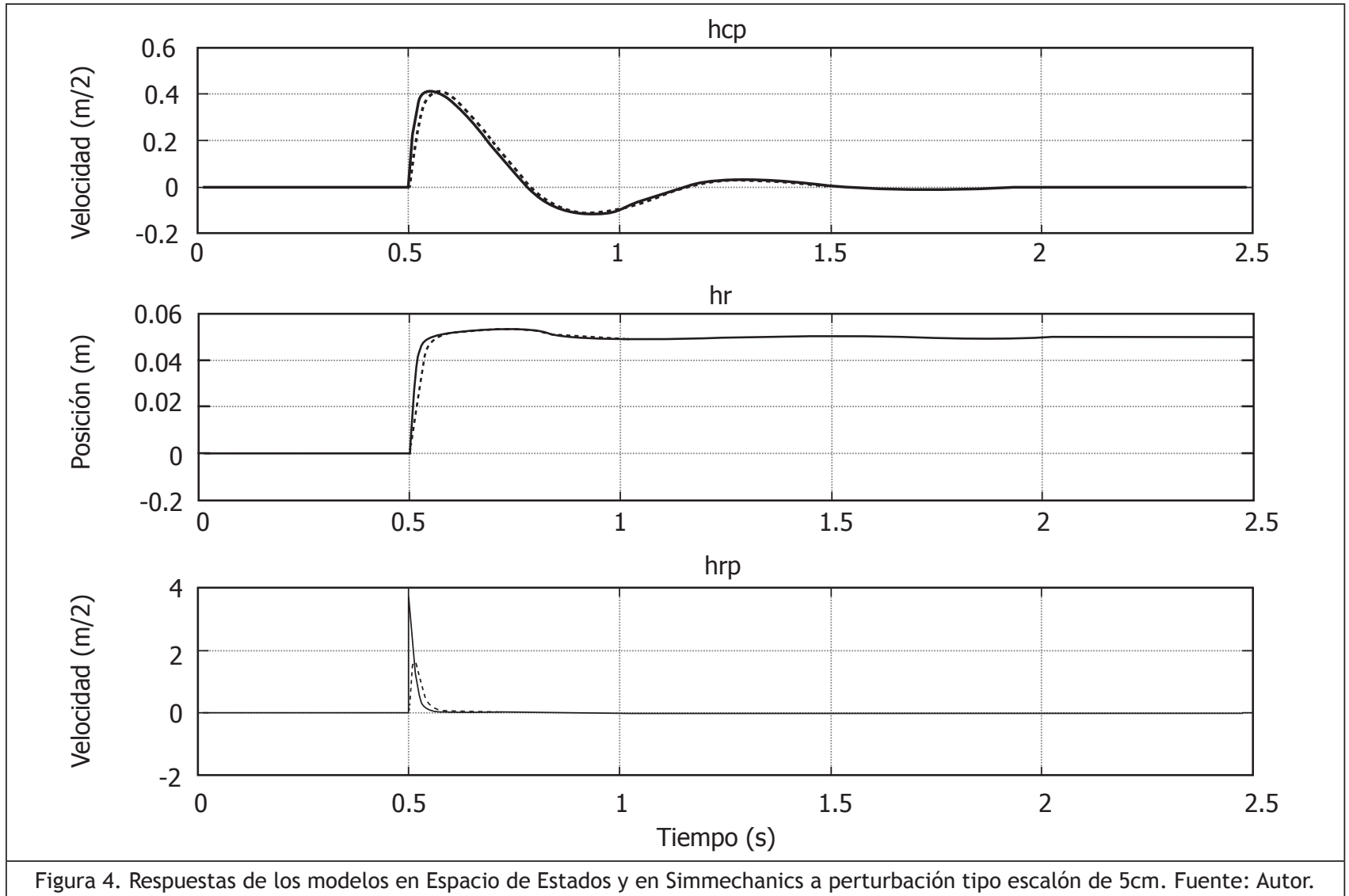

Es posible observar que el comportamiento del sistema modelado en espacio de estados representa con un alto grado de precisión la dinámica del sistema de masas suspendidas, evitando la presencia de errores en el comportamiento del controlador debido a comportamientos no modelados en el sistema en espacio de estados con respecto al compotamiento del sistema simulado en Simmechanics.

En la Figura 5 se ilustra el comportamiento de las variables de posición y velocidad del chasis y la rueda, visualizando simultaneamente la respuesta del sistema pasivo (No controlado) y el sistema activo (Controlado) utilizando MPC.
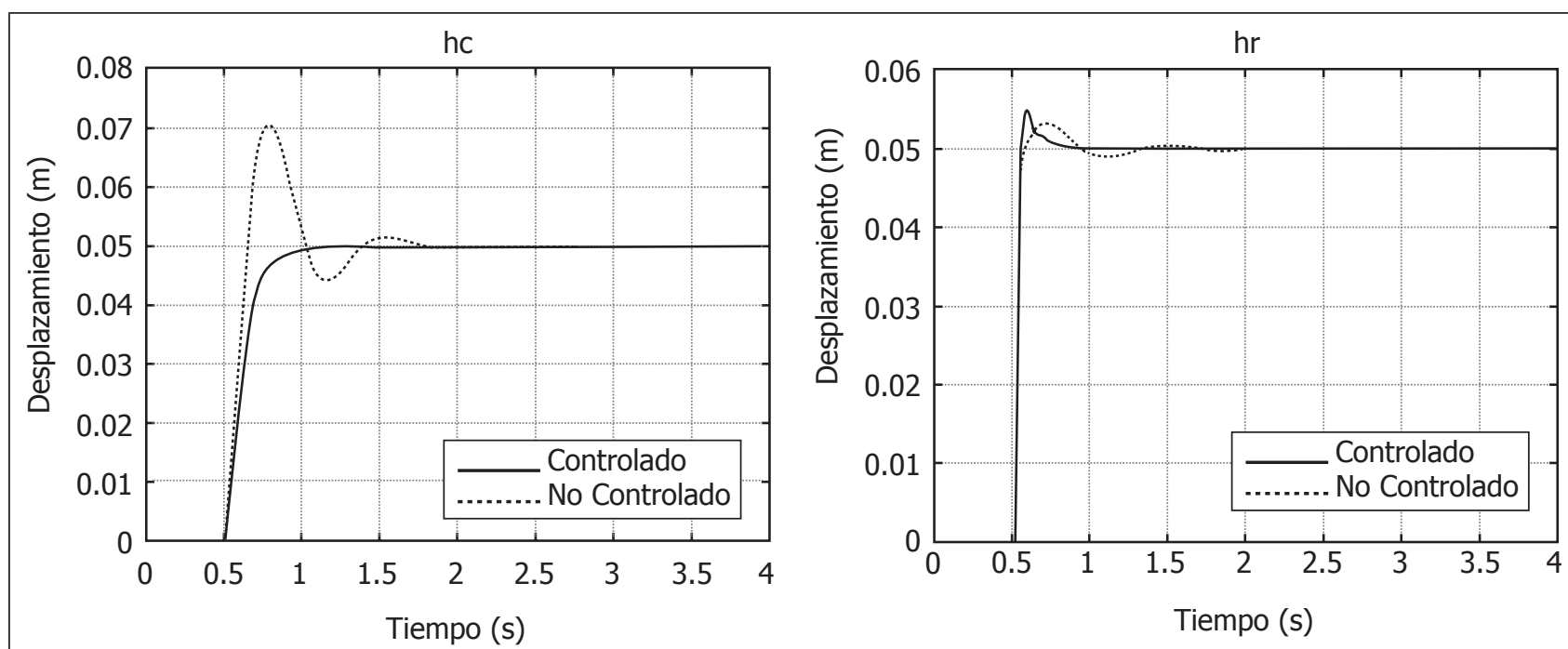

Figura 5. Señales de desplazamiento y velocidad de la masa del chasis y la masa de la rueda. Fuente: Autor. 

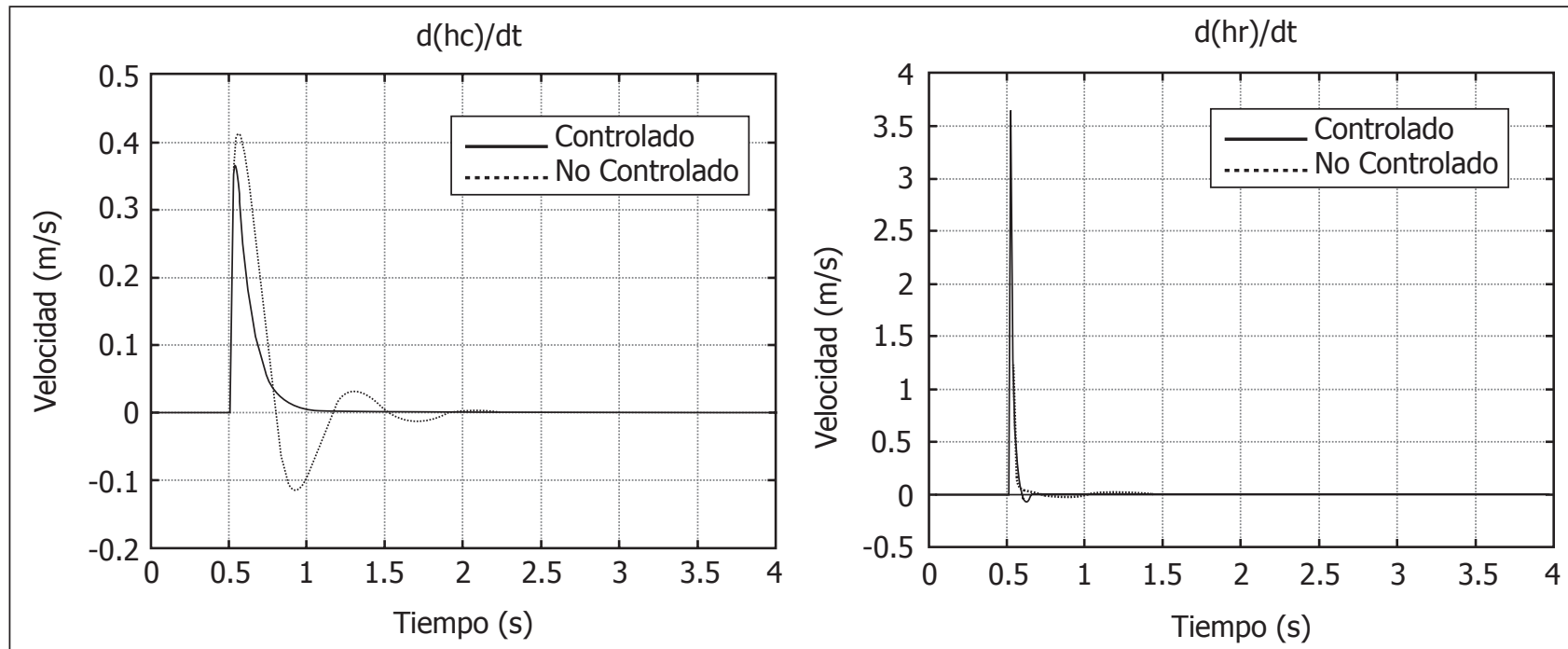

Figura 5. Señales de desplazamiento y velocidad de la masa del chasis y la masa de la rueda. Fuente: Autor.

Las respuestas obtenidas del sistema controlado presentan una reducción significativa en los cambios de velocidad que experimenta el chasis, estabi- lizando más rápidamente el chasis y evitando el exceso de oscilaciones al presentarse una perturbación de $5 \mathrm{~cm}$.

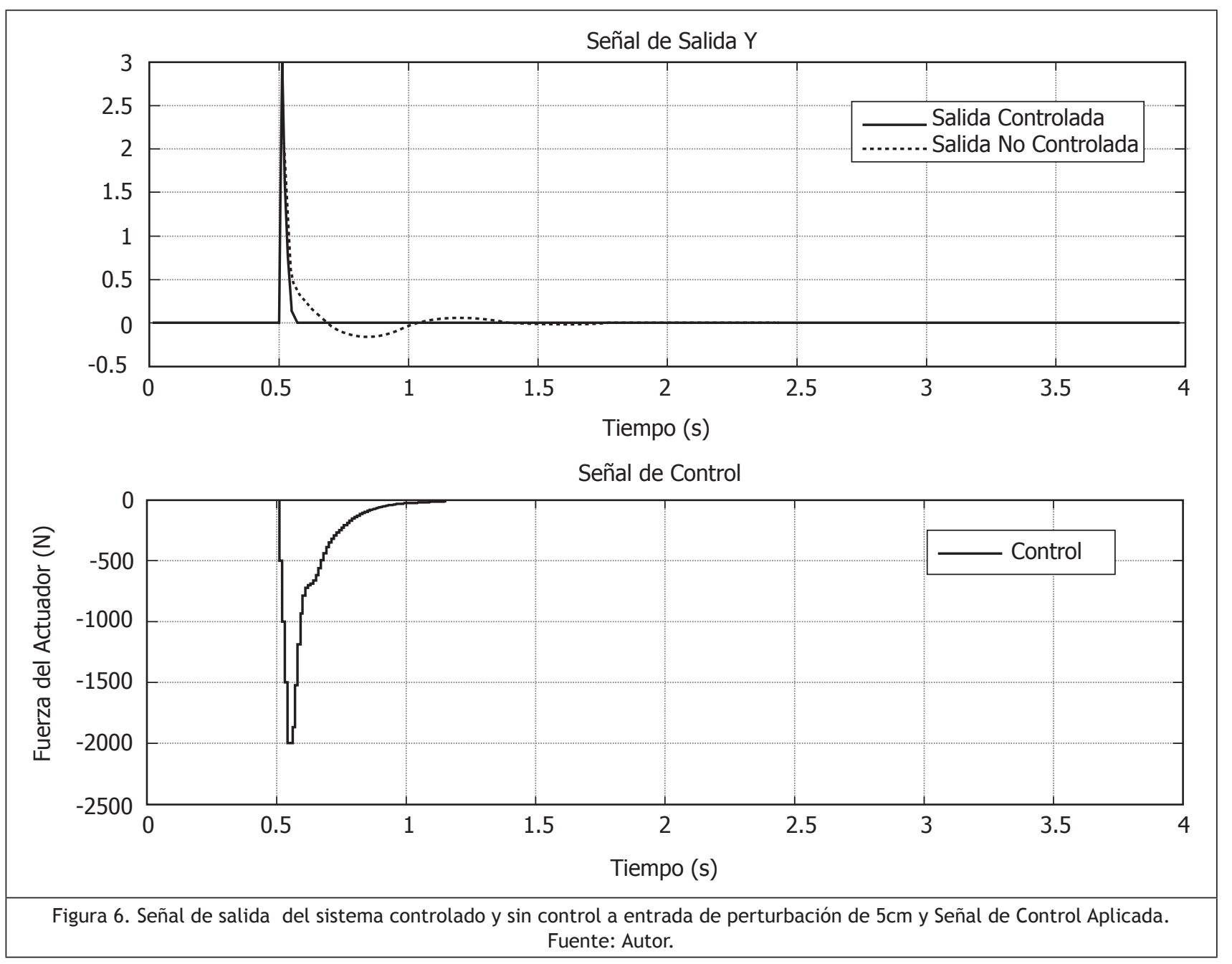


Es posible apreciar como el sistema controlado reduce significativamente la amplitud de las oscilaciones presentes en la señal de salida y el tiempo que tarda el sistema en estabilizarse después de introducirse la perturbación. Adicionalmente se observa como la señal de control obtenida mediante el proceso de optimización con restricciones respeta las restricciones impuestas al actuador, por lo cual no se presentan variaciones superiores a $0.5 \mathrm{KN}$ y el límite de $2 \mathrm{KN}$ no es superado. (Figura 6)

\section{CONCLUSIONES}

El comportamiento del sistema controlado es significativamente superior en reducción a las perturbaciones experimentadas en el chasis, causadas por irregularidades existentes en la superficie de desplazamiento de los vehículos, comprado con la respuesta de la suspensión no controlada. Mediante la sintonización del controlador de manera adecuada es posible eliminar las oscilaciones en la respuesta del sistema, lo cual evita que se alcancen frecuencias de resonancia que podrían incomodar a pasajeros y causar daños en la carga.

La función de salida que se desa controlar puede usarse como parámetro de sintonización del controlador, así como la longitud de los intervalos de predicción y control, con el objetivo de obtener el comportamiento deseado del sistema controlado.

La filosofía de diseño de los controladores predictivos basados en modelo es fácil de comprender si se tienen bases de optimización y conocimiento de modelamiento de sistemas en espacio de estados. Como la técnica no implica el conocimiento profundo de técnicas de control y se fundamenta desde las técnicas de optimización, es posible su aplicación en diferentes tipos de sistemas sin tener que modificar los fundamentos de la misma.

Los controladores predictivos basados en modelo permiten incluir dentro del diseño del controlador las restricciones impuestas por las limitaciones físicas de los elementos que actúan en el sistema real, obteniendo señales de control óptimas que minimizan una función objetivo definida sin violar las restricciones impuestas. En el caso de la suspensión activa vehicular este aspecto es importante, ya que el actuador, la geometría y las limitaciones físicas de los elementos que la componen imponen restricciones que el diseño clásico de controladores no contemplan. Estas características del control MCP aplicadas en los sistemas de suspención vehicular permiten mejorar el desempeño de la suspensión dentro de las limitaciones impuestas, incrementando la seguridad y el confort de pasajeros y carga.

Las diferentes técnicas usadas en el desarrollo de suspensiones activas permiten una mejora en la dinámica del sistema, tal como se aprecia en este caso con el uso de control predictivo. Los resultados obtenidos motivan a la imple-mentación física del sistema, a su difusión y uso en los vehículos comerciales, con el fin de incrementar la seguridad de personas y carga.

\section{BIBLIOGRAFIA}

1. M. S. Fallah, R. B. (2008). New Nonlinear Model of Macpherson Suspension System for Ride Control Applications. IEEE.

2. Keum-Shik Hong, D.-S. J. (1999). A New Modeling of the Macpherson Suspension System and its Optimal Pole-Placement Control. IEEE.

3. Jorge Hurel, A. M. (2012). Nonlinear TwoDimensional Modeling of a McPherson Suspension for Kinematics and Dynamics Simulation. IEEE.

4. Wang, L. (2009). Model Predictive Control System Design and Implementation Using $M A T L A B$. Springer.

5. Ogata, K. (1998). Ingeniería de Control Moderna ( $3^{\mathrm{a}}$ edición ed.). Pearson.

6. Maciejowski, J. M. (2000). Predictive Control whit Constrains. Pearson.

7. Hredzak, B., Agelidis, V. G., \& Jang, M. (2013). A Model Predictive Control System for a Hybrid Battery-Ultracapacitor Power Source. IEEE.

8. Khalid, M. U., \& Kadri, M. B. (2012). Liquid Level Control of Nonlinear Coupled Tanks System using Linear Model Predictive Control. IEEE.

9. Kamal, A. S., Imura, J.-i., Hayakawa, T., Ohata, A. , \& Aihara, K. (2013). Smart Driving of a Vehicle Using Model Predictive Control for Improving Traffic Flow. IEEE.

10. Bart L.J. Gysen, T. P. (03 de 2011). Efficiency of a regenerative direct-drive electromagnetic active suspension. IEEE. 